
\title{
Grow your business, acquire
patients and improve recall effectiveness with iSmile
}

\section{iSmile new

Practice management systems are traditionally seen as a means to store patient data including details of treatments, medical histories and processing payments. As dentists begin to explore opportunities to grow their business, it makes sense to take advantage of the rich data which is already present in your practice. Having the right tools in your practice management system will help you to build a cost effective marketing strategy.

A built-in automated recall campaign manager delivers improved recall effectiveness

Receptionist and practice managers can find it challenging meeting the demands of a busy practice as well as supporting marketing activities through carefully targeted messaging for recalls, reminders and for acquiring new patients. Recalls are often overlooked or sent only once in a cycle, in the hope that the patient receives the message first time. Missed appointment patients can also be overlooked and they end up in an 'idle' state with no activity in the practice. All of these issues lead to large lists of patients that are registered with the practice but are not being treated.

Business intelligence experts at iSmile have thoroughly investigated these issues and iSmile now has a built-in Campaign Manager which can automatically generate up to three rounds of appointment reminders and up to eight rounds of recall reminders. We recognise that each practice is different so our Campaign Manager is highly customisable and practice managers can keep a handle on campaign costs as well as seeing a real return on investment with iSmile's extensive reporting features.

Each message can be set to be sent via any channel (eg SMS, email, letter or patient preference) and the message for each round of reminders or recalls can be customised. With all the activity happening automatically in the background it eases pressure on practice managers and receptionists and also provides detailed information on the state of each registered patient, ensuring that no patient is lost in the system.

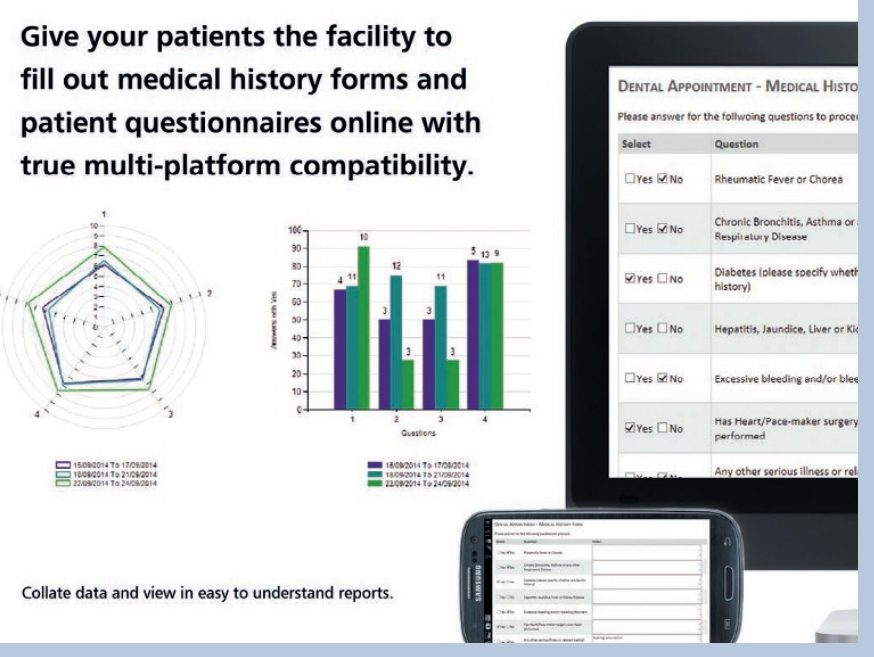

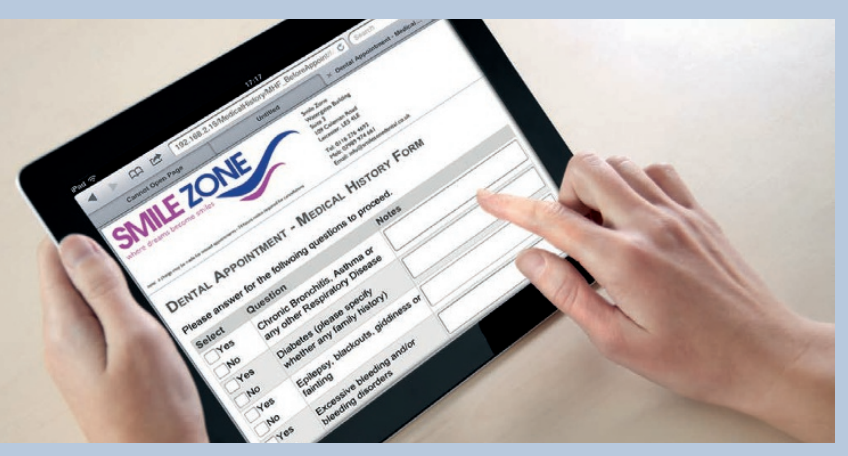

Case study: regular recall reminders generates $15 \%$ increase in bookings

Context: The subject practice was already issuing recall reminders to patients, however the reminders were missing a defined rhythm and the nature of the reminders (SMS, email, letters etc) was sporadic. The practice saw immediate benefits in using the iSmile Campaign Manager.

Benefits realised:

$\checkmark$ Efficiencies in working practices through automated processing $\checkmark$ Improved patient journey through consistent messaging

$\checkmark$ Cost efficiency as more frequent free of charge (email) reminders are generated

$\checkmark$ Increase in bookings and revenue.

\section{The rise of the connected patient}

The digital age has created a connected world with constant access to key information anywhere, anytime on any device. iSmile's Patient Portal can be accessed from any device and is branded to the practice's corporate identity. The secure portal enables patients to complete their medical history ahead of their appointment and minimises surgery wait times, allowing dentists to see more patients during the day. The iSmile Patient Portal also captures patient feedback on their experience which can then be analysed at the practice and also posted on social media platforms. This has proven to be a powerful tool in creating an online identity and acquiring new patients.

iSmile can also be used to understand and react to the needs of a patient. Patient interest for cosmetic treatments such as whitening, veneers and tooth straightening can be recorded. Using these markers the practice is able to develop targeted marketing material and run campaigns for selected treatments.

\section{Big data for small businesses}

In conclusion, the data, processes and workflow present in the practice management system is the best source for marketing. Using a system that automates processes and relieves pressure from practice managers and owners can result in double digit growth in revenues and significantly improve patient perceptions with a more 'connected' experience in the digital age. 